\title{
THE EXPRESSION OF ANTIGENS BY EMBRYONIC NEURONS AND GLIA IN SEGMENTAL GANGLIA OF THE LEECH HAEMOPIS MARMORATA ${ }^{1}$
}

\author{
EDUARDO R. MACAGNO, ${ }^{* 2}$ RANDALL R. STEWART, ${ }^{*}$ AND BIRGIT ZIPSER $\ddagger$ \\ * Department of Biological Sciences, Columbia University, New York, New York 10027 and $\ddagger$ Cold Spring Harbor Laboratory, \\ Cold Spring Harbor, New York 11724
}

Received December 13, 1982; Revised February 28, 1983; Accepted March 28, 1983

\begin{abstract}
Monoclonal antibodies (mAbs) raised against adult leech nervous systems were screened on embryos of the leech Haemopis marmorata in order to determine when in development specific antigens are first expressed and the order in which they are expressed by different cells or tissues. Three of the mAbs produced by Zipser and McKay (Zipser, B., and R. McKay (1981) Nature 289: 549-554) were screened: Lan3-1, Lan3-5, and Lan3-6. Each mAb shows a different pattern of labeling in the adult leech nerve cord (Zipser, B. (1982) J. Neurosci. 2: 1453-1464). The embryonic stages studied were from 5 days after egg deposition to 30 days (emergence from the cocoon). The pattern of labeling was assayed in whole mounts using horseradish peroxidase-conjugated second antibodies. The principal results are as follows. (1) Antigens recognized by Lan3-5 are first expressed by the glia of the roots of the anterior segmental ganglia at 6 to 7 days, several days later by the interganglionic connective glia, and near the end of embryonic development by ganglionic neurons. An anterior to posterior temporal gradient is observed in the expression of these antigens. In addition, Lan3-5 also labels the protonephridia and nephridia from early development onward. (2) Antigens recognized by Lan3-6 are first expressed by a pair of neurons in each segmental ganglion and later in development by additional neurons. By the time of emergence, however, only about half of the neurons that label in the adult have done so, implying that some neurons express these antigens postembryonically. Labeling with Lan3-6 is first seen in neuronal somata and only later in neuronal processes. (3) Antigens recognized by Lan3-1 and expressed by segmentally specific neurons in ganglia 5 and 6 are not detectable during embryonic development, but are so at early postembryonic stages. Thus, these three mAbs provide an approach to study different aspects of the development of the leech nervous system, specifically the relation between glial and neuronal differentiation and the genesis of segmentally specific phenotypes.
\end{abstract}

The recent demonstration by Zipser and McKay (1981) that, among monoclonal antibodies (mAbs) raised against whole adult leech central nervous system (CNS), some label identified cells or small groups of cells, provides direct evidence for the existence of individual molecular signatures in adult leech neurons and glial cells. Little is currently known about the roles of the antigens bound by these mAbs in establishing the structure, function, or synaptic connectivity of these cells. Understand-

\footnotetext{
${ }^{1}$ We thank Carol Schley for her invaluable technical assistance and Murray Flaster for his careful reading of the manuscript. This work was supported by National Institutes of Health Grants NS 14946 (E. R. M.) and NS 17984 (B. Z.), by National Science Foundation Grant BNS 78-248-72 (B. Z.), and by a Whitehall Foundation grant.

${ }^{2}$ To whom correspondence should be addressed, at Department of Biological Sciences, 1003 B Fairchild, Columbia University, New York, NY 10027.
}

ing these roles requires information about the identity, localization, and temporal expression of these antigens. Initial efforts to identify them and to determine their distributions within cells have been reported recently (Zipser et al., 1981, 1982; McKay et al., 1982; Hockfield and McKay, 1983). We have begun to examine when during the life of the animal these antigens first appear, and what the degree of cell differentiation is when the antigens are first expressed.

A practical reason also stimulates our interest in studying the developmental pattern of labeling with the mAbs raised to adult tissues. Whereas particular neurons can be relatively easy to identify in an adult nervous system on the basis of specific morphological and electrophysiological criteria, such identification may be difficult, if not impossible, during neuronal ontogenesis. The reasons for this are many. For example, size is often used to distinguish a cell from its neighbors, but at early 
developmental stages neurons are frequently similar in size. Furthermore, the characteristic shape and electrical properties may be very different in embryonic and adult stages. Markers that distinguish individual ncurons from each other as well as from non-neuronal cells would thus be an invaluable aid in the study of the early stages of the differentiation of these neurons. To the extent that neurons express individual molecular signatures early in their development, antibodies raised against such molecules can play a useful role as identifying markers.

The adult leech CNS consists of a head ganglion (with supra- and subesophageal sections), 21 segmental ganglia (SG), and a tail ganglion. Paired lateral connective nerves, along with an unpaired medial one (Faivre's nerve), run along the nerve cord from the head to the tail ganglia. The SG are connected to the periphery by pairs of bilateral nerve roots. During development 32 ganglionic primordia are made. The four most anterior fuse to form the subesophageal section of the head ganglion, the seven most posterior fuse to form the tail ganglion. The $21 \mathrm{SG}$ are quite similar to one another, each containing about 400 neurons, with the exception of those in the 5th and 6th body segments, which contain a few hundred more cells in hirudinid leeches (Macagno, 1980).

The work reported here was carried out on the hirudinid leech Haemopis marmorata. The general development of hirudinid leeches has been described by many authors (reviewed in Dawydoff, 1959 and Mann, 1962). A recent report by Fernandez and Stent (1983) describes in some detail the formation of the germinal plate and CNS of Hirudo medicinalis. Our observations on Haemopis marmorata indicate that these aspects of development are essentially identical in these two species.

We have examined the pattern of labeling of the $\mathrm{SG}$ of $H$. marmorata at different developmental stages by mAbs raised against adult nerve cords. The mAbs used (Lan3-5, Lan3-6, and Lan3-1) are among those first described by Zipser and McKay (1981). We selected these three mAbs because in the adult they recognize antigens with very different distributions (Zipser, 1982). Lan3-5 labels glial cells in the connectives and roots and between 10 and 20 neurons in each SG. With Lan3-5 we can study the development of central and peripheral fiber tracts and the relative time of expression of antigens by neurons and glial cells. The second mAb, Lan3-6, labels about $10 \%$ of the neurons in each SG in the adult, and thus we can ask whether all neurons that express the antigens recognized by this $\mathrm{mAb}$ do so at the same time in development and, ultimately, whether they do so at a specific point during their differentiation. Lan3-1 labels a pair of small neurons in each SG and an additional pair of larger neurons in ganglion 5 and ganglion 6 , which innervate the sexual organs. Using this $\mathrm{mAb}$ we can ask when in development this segmental specialization occurs. The results presented in this paper answer these and other questions. Some of these results have been reported previously (Stewart et al., 1982).

\section{Materials and Methods}

Large, gravid Haemopis marmorata were placed in plastic boxes containing a mixture of potting soil and milled sphagnum moss. The boxes were checked every other day for cocoons containing embryos. Once found, the cocoons were placed either in $100-\mathrm{mm}$ Petri dishes or in $100-\mathrm{ml}$ plastic cups and covered with moist soil. These were maintained in an incubator at $23 \pm 1^{\circ} \mathrm{C}$.

Embryos of various ages were selected for dissection and fixation. Generally, a cocoon was immersed in cold saline (115 mM NaCl, $1.8 \mathrm{mM} \mathrm{CaCl}, 1.8 \mathrm{~mm} \mathrm{MgCl}, 4.0$ $\mathrm{mm} \mathrm{KCl}$, and $10 \mathrm{~mm}$ Tris maleate adjusted to $\mathrm{pH} 7.4$ with $\mathrm{NaOH}$ ), one end was sliced off with a razor blade, and the embryos were removed. While in saline, each embryo's yolk was removed and the embryo was pinned out dorsal side up in Sylgard-covered dishes. Adult nerve cords to be used as controls were also dissected and pinned out in Sylgard-covered dishes. Both embryos and adult nerve cords were fixed at room temperature in Bouin's fixative for $4 \mathrm{hr}$ or in $4 \%$ paraformaldehyde in $0.1 \mathrm{M}$ phosphate buffer for $30 \mathrm{~min}$. After fixation they were removed from the dishes and were either acetone extracted for $7 \mathrm{~min}$ or dehydrated and xylene extracted for $1 \mathrm{~min}$, then stored in phosphate-buffered saline (PBS) containing 1 drop of $10 \%$ sodium azide/5 ml or incubated in $\mathrm{mAb}$ as described below.

Fixed and extracted tissues were placed in $\mathrm{mAb}$ supernatant or ascites fluid (dilution of 9 parts supernatant to 1 part $20 \%$ Triton $\mathrm{X}$ and of 1 part ascites to 1000 parts PBS/2\% Triton X). After overnight incubation with $\mathrm{mAb}$, the tissue was rinsed for 5 min with $\mathrm{PBS} / 2 \%$ Triton $\mathrm{X}$, incubated 2 to $4 \mathrm{hr}$ in horseradish peroxidaseconjugated goat anti-mouse IgG (heavy and light chain specific; N. L. Cappel Laboratories), and rinsed again with PBS/2\% Triton $\mathrm{X}$ for 5 min. Next, the tissue was repinned in Sylgard dishes, rinsed briefly in $0.05 \mathrm{M}$ Tris buffer, and incubated in $20 \mathrm{mg}$ of diaminobenzidine $/ 5$ $\mathrm{ml}$ of $0.05 \mathrm{M}$ Tris buffer for 5 to $15 \mathrm{~min}$, then, 1 drop of $1 \% \mathrm{H}_{2} \mathrm{O}_{2}$ was added, and the reaction was allowed to proceed until the tissue became light brown. Lastly, the tissue was dehydrated in an ethanol series, cleared in xylene, and mounted using Permount between two coverslips.

The degree and the reproducibility of labeling with each $\mathrm{mAb}$ were dependent on the type of fixative used. For example, Lan3-1 only labeled tissues fixed in Bouin's, whereas Lan3-6 labeled optimally when paraformaldehyde was used. Lan3-5, however, worked equally well with either method of tissue fixation. The embryonic and adult labeling patterns were always obtained for each $\mathrm{mAb}$ using the same procedures.

mAb staining patterns were analyzed in whole mounts under Nomarski optics using a computer-coupled light microscope (described in Macagno, 1980). The outline of a ganglion and the perimeter of each stained neuron were drawn into the computer to determine the number and spatial distribution of neurons labeled by each $\mathrm{mAb}$. For Lan3-6, the labeled cells in each SG were counted twice, once each by different investigators, and then averaged to obtain the values quoted in this report. Nomarski optics were also used to photograph the embryos and to study their morphological features to assess their relative ages.

\section{Results}

The observations reported below were made for the most part on embryos from 5 days old to emergence, 

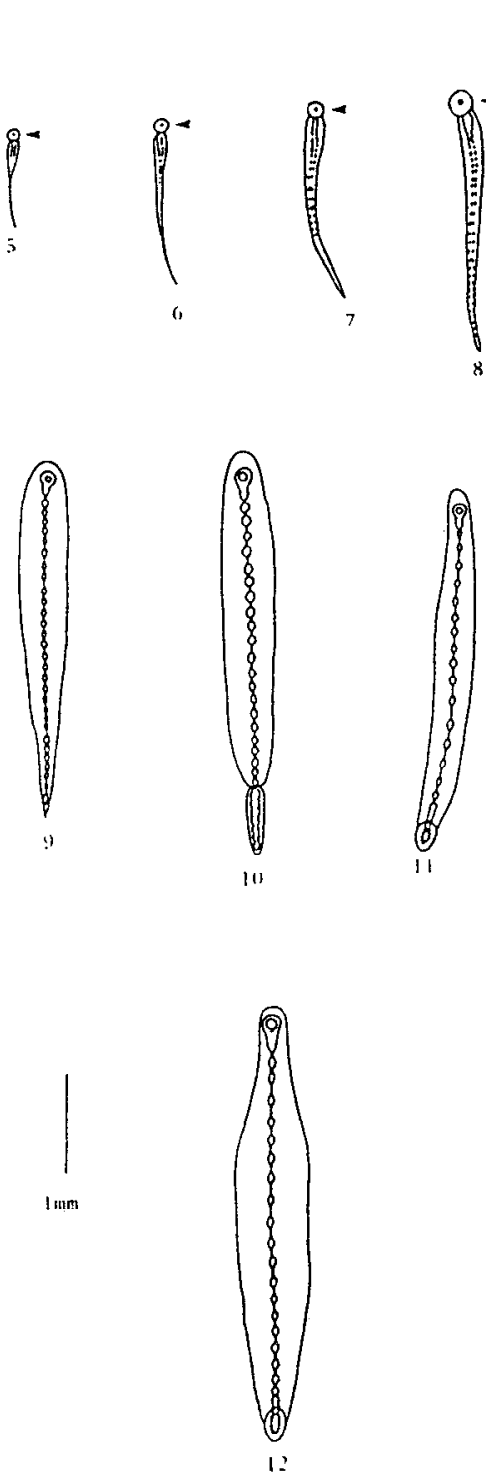
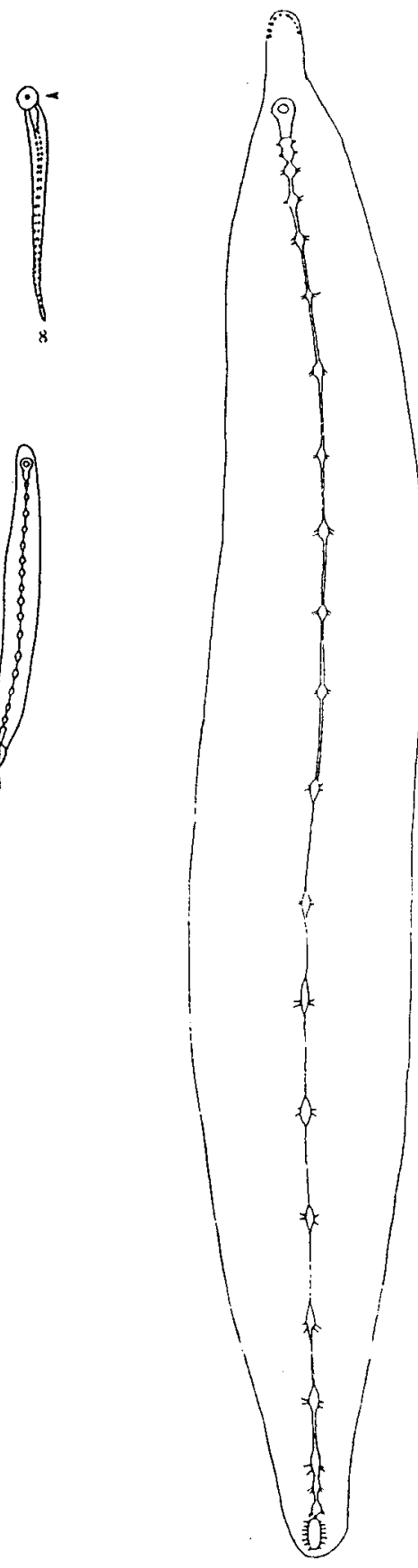

25 which occurs about 30 days after egg deposition at temperatures of 22 to $24^{\circ} \mathrm{C}$. The general level of development of the nervous system at different embryonic stages is described in Figure 1. Comparison of the developmental staging scheme proposed by Stent et al. (1982) for the embryonic development of the glossiphoniid leech Helobdella triserialis with our own observations on the hirudinid Haemopis marmorata, suggests to us that the embryonic stages we have studied correspond to those that these authors labeled stages 8 through 11 . Although the early developmental stages in these two leech orders are very different (Dawydoff, 1959; Mann, 1962), it is probably the case that later stages, and particularly the formation of the CNS, are fairly similar (Fernandez and Stent, 1983). In the studies with Lan3-1, some early postembryonic stages were also examined.

The pattern of label obtained with Lan 3-5. In the adult CNS, Lan3-5 has been shown to bind to glial components of the roots and connectives and to a small number of neurons (10 to 20 ) in each SG, of which two pairs are the pressure mechanosensors ( $\mathrm{P}$ cells).

The first structures in the embryonic CNS that are labeled by Lan 3-5 are the ganglionic roots. Little labeling is detected in 6 to 7-day embryos (Fig. 2, $a$ and $b$ ), even in anterior segments. At later stages (Fig. 2, $c$ and $d$ ), more posterior roots become labeled and those that are anterior are labeled more intensely. In addition, label extends further along the roots toward the body wall, and a cell body lying between the roots at the ganglionic margin (probably of the glial cell of the roots) becomes intensely labeled (see Fig. 3, $a$ and $b$ ). The pattern of labeling indicates that Lan3-5 is binding to a cytoplasmic component of the glia that ensheath the neuronal processes in the root nerves. A temporal gradient of maturation from the older anterior segments to the younger

Figure 1. Drawings of Haemopis embryos of various ages. The numbers under each drawing refer to the number of days after cocoon deposition. Only the area of the germinal plate has been drawn; the larval organs are not shown, with the exception of the first four stages, where the larval mouth is indicated by an arrowhead. All the ganglionic primordia are visible by 8 days. 'I'he four ganglia that form the subesophageal ganglion begin to fuse at 8 days, the seven that form the tail ganglion begin to fuse at 10 days. The tail sucker begins to form at 10 to 11 days. Anterior is up.

Figure 2. Patterns of labeling with Lan3-5 in whole mounts of various embryonic stages. In all cases anterior is up. $a$, Low power micrograph of a 6- to 7-day embryo. The picture extends from the first SG (1, partially cut off at the top) to the ninth SG (9, partially cut off at the bottom). The forming nephridia are indicated on the left side by arrowheads. Labeling with Lan3-5 can be found in the four most anterior nephridia. Bar $=100 \mu \mathrm{m}$. $b$, Higher magnification micrograph of a region of $a$. The region includes SG1 to SG4 ( 1 to 4 ). Labeling with Lan3-5 can be seen in the nephridia $(N)$ and in the lateral margins of the first ganglion, where the root glia are beginning to form $(r)$. Longitudinal processes are indicated by arrowheads in ganglia 3 and 4 . The ganglia lie adjacent to one another, and the connectives have not formed. Bar $=50 \mu \mathrm{m}$. $c$, Micrograph of a central region (SG8 to SG12) in a 12-day embryo. The nephridia $(N)$ are heavily labeled by Lan3-5, as are the ganglionic roots $(r)$. The interganglionic connectives $(c)$ are visible, and the ventral blood sinus $(b s)$ is beginning to form. Magnification is the same as in a. $d$, Micrograph of the posterior region of a 13-day embryo, including SG13 through SG20 (only 13 and 20 are numbered). The labeled nephridia are seen on each side of the nerve cord. The ganglionic roots are labeled in all ganglia, but label extends further toward the periphery in the more anterior ganglia. $B a r=100 \mu \mathrm{m}$. 

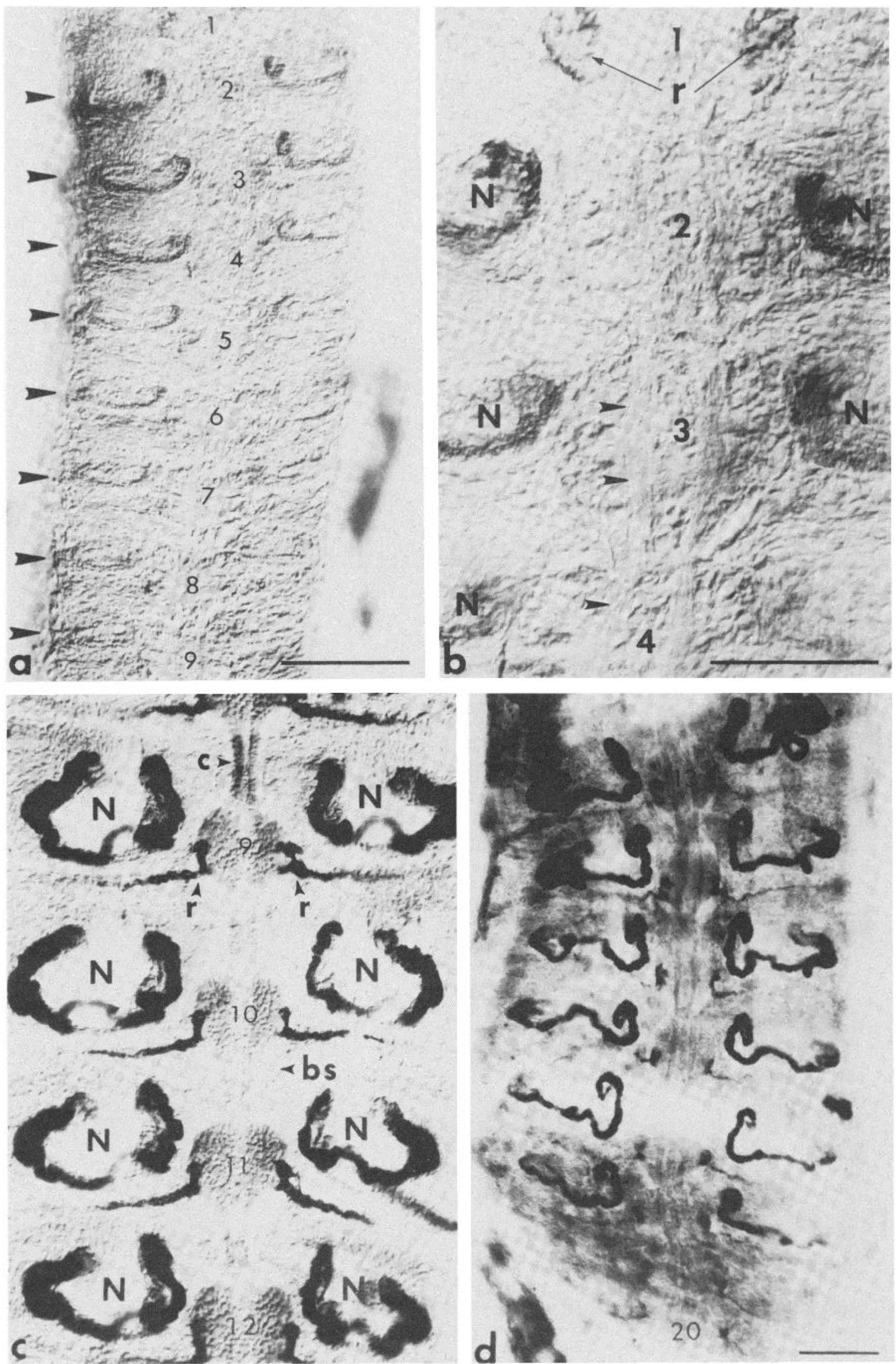

Figure 2 

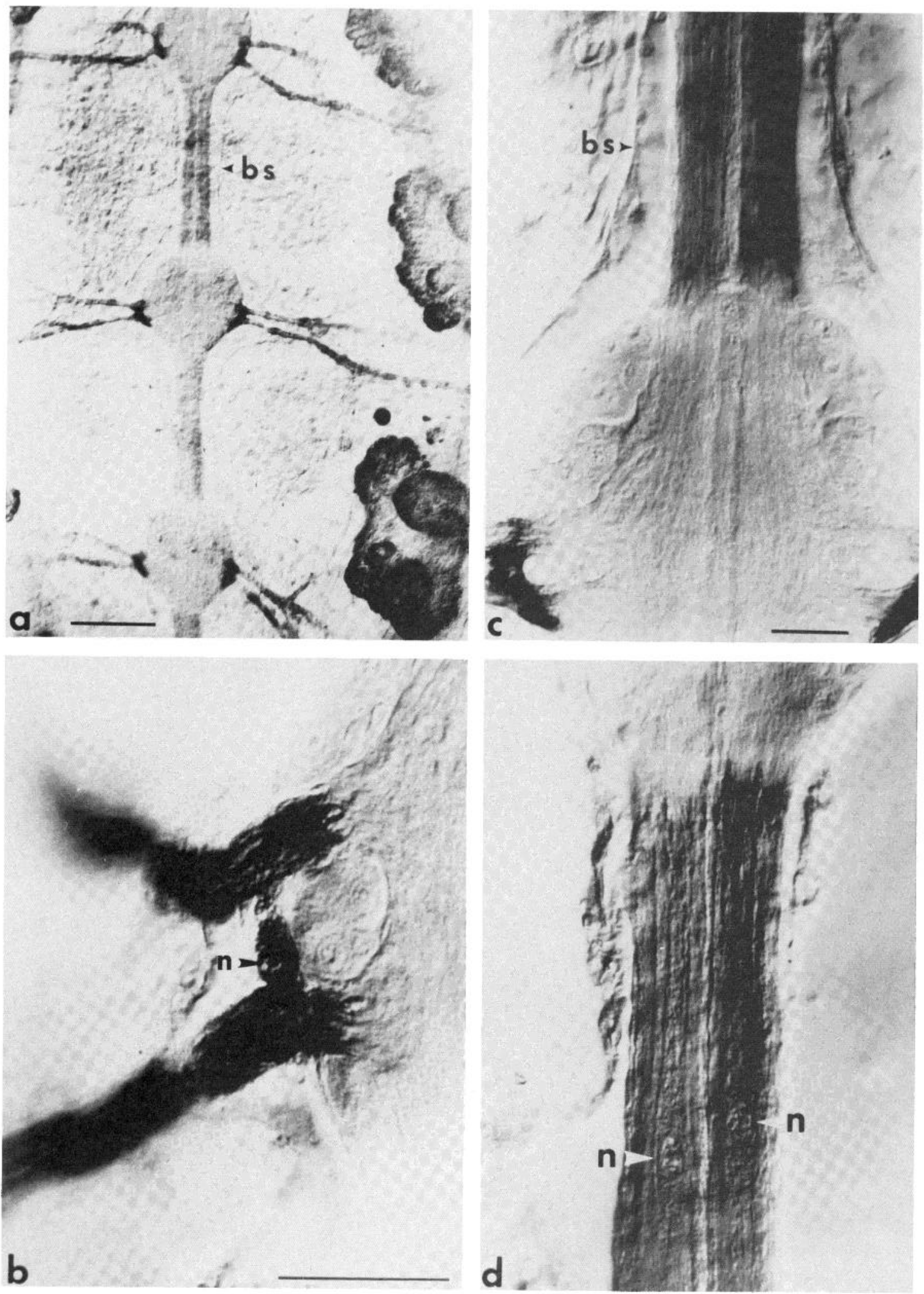

Figure 3. Labeling with Lan3-5 in a whole mount of a 25-day embryo. Anterior is up in all micrographs. $a$, Low power micrograph showing SG15 to 17 . The roots are labeled, as are the nephridia on the right. The blood sinus $(b s)$ is well formed at this stage and contains blood. Bar $=100 \mu \mathrm{m}$. $b$, Detail of the roots of SG9. The labeled cell between the roots contains a nucleus (n). Bar $=20 \mu \mathrm{m} . c$, SG15 and a portion of the connective anterior to the ganglion. The connective and the roots are heavily labeled, but neurons are not. $b s$ indicates the blood sinus. Bar $=20 \mu \mathrm{m}$. $d$, Region of the labeled connective posterior to SG12. The glial nuclei are indicated $(n)$. Magnification is the same as in $c$. 
posterior ones is apparent. It appears, therefore, that Lan3-5 binds to an antigen which is expressed early in the differentiation of the root glia, at a time when the initial connections between the SG and the periphery are being made.

In contrast to the roots, binding of I Lan3-5 to the connectives is not detectable until about 25 days of development (Fig. 3, $c$ and $d$ ), more than 2 weeks after neuronal processes are first seen in the connectives. The distribution of label, particularly where the connectives enter a ganglion, indicates that Lan3-5 is labeling the two giant glia which ensheath the neuronal processes between each pair of adjacent SG. Since these glia can be seen considerably earlier in development than 25 days (we first see them in whole mounts at 11 to 12 days), it would seem that expression of the antigens bound by Lan3-5 occurs at relatively different stages in the differentiation of the connective and root glia. In comparison, the six packet glia and the two neuropil glia are clearly seen in each SG by the end of embryogenesis, but show no evidence of labeling with Lan3-5, in agreement with the lack of such labeling in the adult.

Labeling of neuronal somata by Lan3-5 is not detected until late in embryogenesis, between 25 and 30 days, just prior to hatching. Even then, labeling can only be seen clearly in some cell bodies in the supraesophageal ganglion, and only faintly, if at all, in a few cells in the SG. By this time P cells can be identified by size and location, but only in a few SG do we detect any binding of Lan35 to these neurons. Thus, the neuronal Lan3-5 antigens, be they identical to or different from the glial antigens, are for the most part expressed at a detectable concentration postembryonically.
In addition to labeling nervous tissue, Lan3-5 labels the embryonic protonephridia and nephridia. Labeling of the nephridia was first observed in 6- to 7-day embryos, soon after the nephridial primordia are apparent under Nomarski optics (see Fig. 2, $a$ and $b$ ). Labeling is stronger in the more anterior segments, disappearing gradually more posteriorly, although nephridial primordia are visible (Fig. 2a). At later stages (8 to 9 days), the label extends further posteriorly and the anterior nephridia are more convoluted. By 11 to 12 days, all nephridia are labeled (Fig. 2, $c$ and $d$ ). The binding of Lan35 to the nephridia of different body segments is a useful marker of embryonic stages, particularly between 6 and 12 days of development. We do not know at present whether neural and nephridial antigens are identical or not.

The pattern of label obtained with Lan3-6. In the adult, Zipser (1982) reported finding that approximately $10 \%$ of the nearly 400 neurons in each SG label with Lan3-6. Some cells label heavily, others lightly. In order to present a quantitative comparison of the adult and embryonic staining patterns, we counted the neurons stained with Lan3-6 in the SG of two adult leeches. The data are presented in the last column of Table I. An example is shown in Figure 4. Some variability is found from preparation to preparation. To what extent this is a consequence of real differences in the cxpression of the antigen or antigens bound by Lan3-6 is not known.

Examples of the labeling of ganglia by Lan3-6 at different embryonic stages appear in Figures 5 to 7 . Counts of numbers of cell bodies labeled as a function of embryonic age are presented in Table I. We first find labeling by Lan3-6 at 8 days of development in a pair of

TABLE I

Numbers of neurons labeled by Lan3-6 in each segmental ganglion (SG) al various limes during embryogenesis and in adulls

The times are given as days after cocoon deposition (e.g., $8 \mathrm{~d}$ is 8 days). For each time, a number $(n)$ of embryos was examined. The data for each SG are the average of separate counts by two investigators for all specimens at each time, rounded off to the nearest one-half.

\begin{tabular}{|c|c|c|c|c|c|c|c|c|}
\hline \multirow{2}{*}{$\begin{array}{l}\text { Segmental } \\
\text { Ganglion }\end{array}$} & \multicolumn{7}{|c|}{ Days of Development } & \multirow{2}{*}{$\begin{array}{c}\text { Adul } \\
(2)\end{array}$} \\
\hline & $\begin{array}{l}8 \mathrm{~d} \\
(3)\end{array}$ & $\begin{array}{l}9 \mathrm{~d} \\
(3)\end{array}$ & $\begin{array}{l}10 \mathrm{~d} \\
\text { (1) }\end{array}$ & $\begin{array}{c}11 \mathrm{~d} \\
(2)\end{array}$ & $\begin{array}{c}12 \mathrm{~d} \\
(3)\end{array}$ & $\begin{array}{c}13 \mathrm{~d} \\
(1)\end{array}$ & $\begin{array}{c}25 \mathrm{~d} \\
(3)\end{array}$ & \\
\hline 1 & 2 & 2 & & 7 & & 16.5 & & 34.5 \\
\hline 2 & 2 & 2 & 2 & 8 & 16 & 19.5 & & 35.5 \\
\hline 3 & 2 & 2 & 2.5 & 8 & 18 & 16 & 19 & 38 \\
\hline 4 & 1.5 & 2 & 2 & 9.5 & 15.5 & 18 & 20 & 36 \\
\hline 5 & 2 & 2 & 2 & 8 & 13 & 19 & 16 & 39 \\
\hline 6 & 2 & 2 & 2.5 & 7.5 & 12 & 20.5 & 22.5 & 38 \\
\hline 7 & 1 & 2 & 2 & 8 & 15 & 26 & 18 & 50.5 \\
\hline 8 & 0 & 2 & 2 & 5.5 & 15.5 & 21 & 25 & 54 \\
\hline 9 & 1 & 2 & 2.5 & 7 & 15 & 21.5 & 22 & 50 \\
\hline 10 & 0 & 1 & 2.5 & 7 & 12 & 18.5 & 21 & 47 \\
\hline 11 & 0 & 1 & 1.5 & 5.5 & 10 & 17 & 17 & 51 \\
\hline 12 & 0 & 1 & 2.5 & 4.5 & 10 & 17.5 & 23 & 47 \\
\hline 13 & 0 & 0.5 & 2.5 & 4 & 9 & 17.5 & 28 & 41 \\
\hline 14 & 0 & 0 & 3.5 & 3.5 & 10 & 15.5 & 17 & 46 \\
\hline 15 & 0 & 0 & 1.5 & 4 & 8 & 17 & 16.5 & 48 \\
\hline 16 & 0 & 0 & 2.5 & 4 & 9 & 16.5 & & 44.5 \\
\hline 17 & 0 & 0 & 2 & 2 & 7 & 14.5 & 15 & 43 \\
\hline 18 & 0 & 0 & 1 & 2.5 & 6 & 17.5 & 15 & 47 \\
\hline 19 & 0 & 0 & 0.5 & 2 & 4 & 13.5 & 20 & 41 \\
\hline 20 & 0 & 0 & 0 & 1 & 2 & 10 & 15 & 35 \\
\hline 21 & 0 & 0 & 0 & 1 & 1.5 & 9 & 13.5 & 31 \\
\hline
\end{tabular}



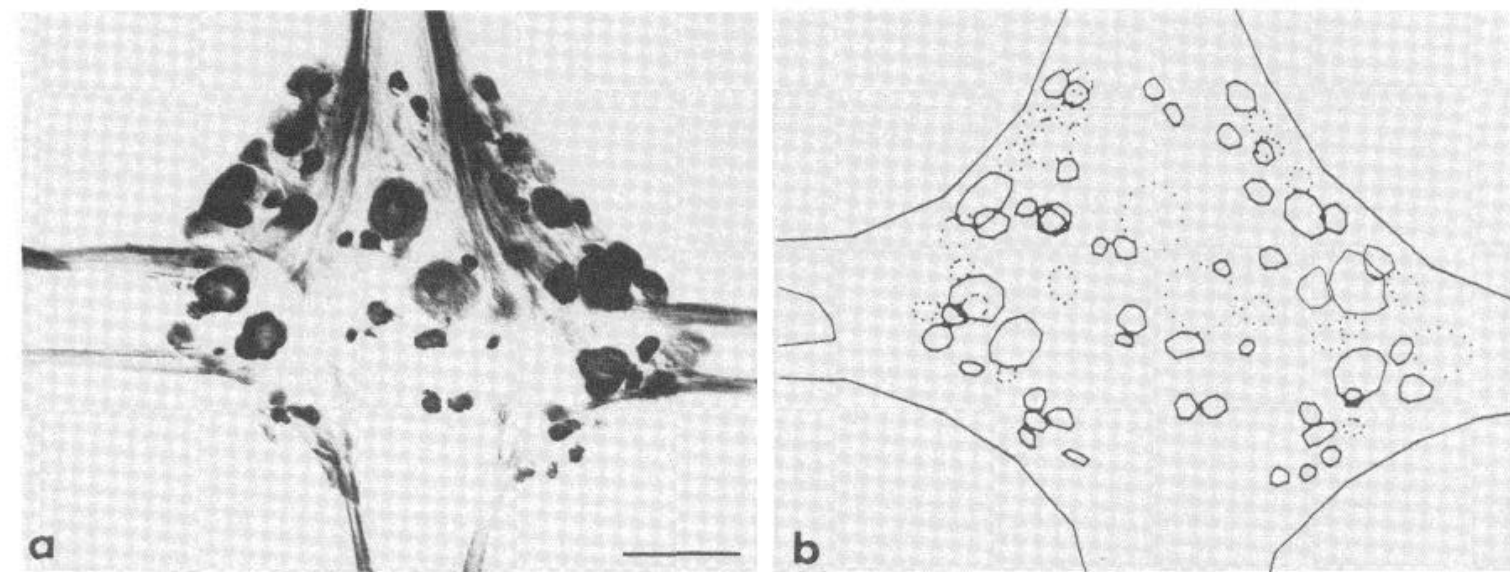

Figure 4. Labeling of an adult SG8 with Lan3-6. a, Micrograph of a whole mount. Many processes are labeled in the connectives, roots, and neuropil. Anterior is up. Bar $=100 \mu \mathrm{m} . b$, Computer-aided drawing of the ganglionic boundary and the labeled neurons. Heavily labeled cells are drawn with solid lines, more lightly labeled ones are drawn with dotted lines.

ventrally located cells (see Fig. 5). A pair of cells in approximately the same location is labeled in the first 6 to 10 ganglia in 8-day animals, and as development proceeds, a similar pair labels in more posterior ganglia, until 11 days, when essentially all ganglia show this pair.

Additional cell bodies begin to label with Lan3-6 at 11 days. These appear initially in the anterior ganglia and gradually, later, in those more posterior (see Table I). The next cells to label are a pair of dorsal cells, followed by other dorsal and ventral cells (see Figs. 6 and 7). Although neurons have not been identified directly in any embryos, it is possible to tentatively identify by size and position the Retzius and the $\mathrm{P}$ cells, which often label with Lan3-6 in the adult. We see only very light, if any, labeling of these neurons even at the latest embryonic stages we have examined.

In order to visualize more easily anteroposterior differences in labeling with Lan3-6, the average numbers of labeled cell bodies per ganglion for four groups of segmental ganglia were calculated. These averages, along with the averages for all ganglia, are given in Table II for each developmental stage and for adults. They are also presented graphically in Figure 8 . The average number of labeled neurons per ganglion rises by 13 days of development to values that remain approximately constant until the end of embryogenesis. These values are only about one-half of the adult values and, therefore, additional neurons must label postembryonically. Differences along the anteroposterior axis are also apparent in Figure 8. For example, the average numbers of labeled neurons in the most posterior SG remain below the overall average throughout development, a characteristic which also prevails in the adult. The SG in midbody regions (SG6-10, SG11-15) begin to label with Lan3-6 after the most anterior ones (SG1-5) do. However, by 25 days they show more labeled neurons than do the anterior ganglia, as they do in the adult (see Table II).

The labeling of cell bodies with Lan3-6 is followed within a few days by labeling of processes in the ganglionic neuropils (see Fig. 6). By focusing through a ganglion, the first processes to label in the neuropil can be followed to the cell bodies of the neurons that labeled earliest in that ganglion. As development proceeds, la- beled processes are also found in connectives and roots (see Fig. 7), and more processes are labeled in the neuropils.

The pattern of label obtained with Lan3-1. In the adult, Lan3-1 labels a pair of posterior dorsal cells in each SG, an additional pair of lateral ventral cells in ganglia 5 and 6 , and processes and varicosities in the ganglionic neuropils whose cells of origin are unidentified (Zipser, 1982).

The youngest embryos in which binding of Lan3-1 was detected were 11 to 12 days old. A pair of dorsal cell bodies, located near the posterolateral margins of each SG, were labeled, along with a few short processes and varicosities in the central region of the neuropil. No binding of Lan3-1 to processes of the labeled neurons was detected. The bound Lan3-1 was distributed within the cell bodies in a non-uniform manner, being more concentrated in the region surrounding the cell nucleus. No additional cells were found to be labeled in either SG5 or SG6. At later embryonic stages more processes and varicosities in the neuropil, some nerve fibers in the connectives, and the initial segments of the processes of the labeled pairs of neurons were found to bind Lan3-1. However, in no embryonic stage up to 25 days did we find additional cells in SG5 or SG6 with detectable binding of Lan3-1. The locations of the labeled embryonic cells are similar to those of the cells common to all SG described by Zipser and McKay (1981) for the adult nerve cord. The labeled cells in SG6 from a 20-day embryo can be seen in Figure 9.

In order to determine whether the lack of Lan3-1 labeling of the extra neurons in SG5 and SG6 is due either to absence of the cells before hatching or to their failure to express the antigens bound by Lan3-1, we counted the cells in individual SG at various embryonic and a few early postembryonic stages. Preliminary data on SG5, SG6, SG7, and SG10 from a few specimens indicate that cell numbers in each of these ganglia are approximately the same in the embryo. At 12 days there are about 440 cells per SG, at 20 days only about 395 . Since the adult number of neurons in SG7 and SG10 is about 400 (Macagno, 1980), it would appear that the adult value is achieved at late embryonic stages, although 

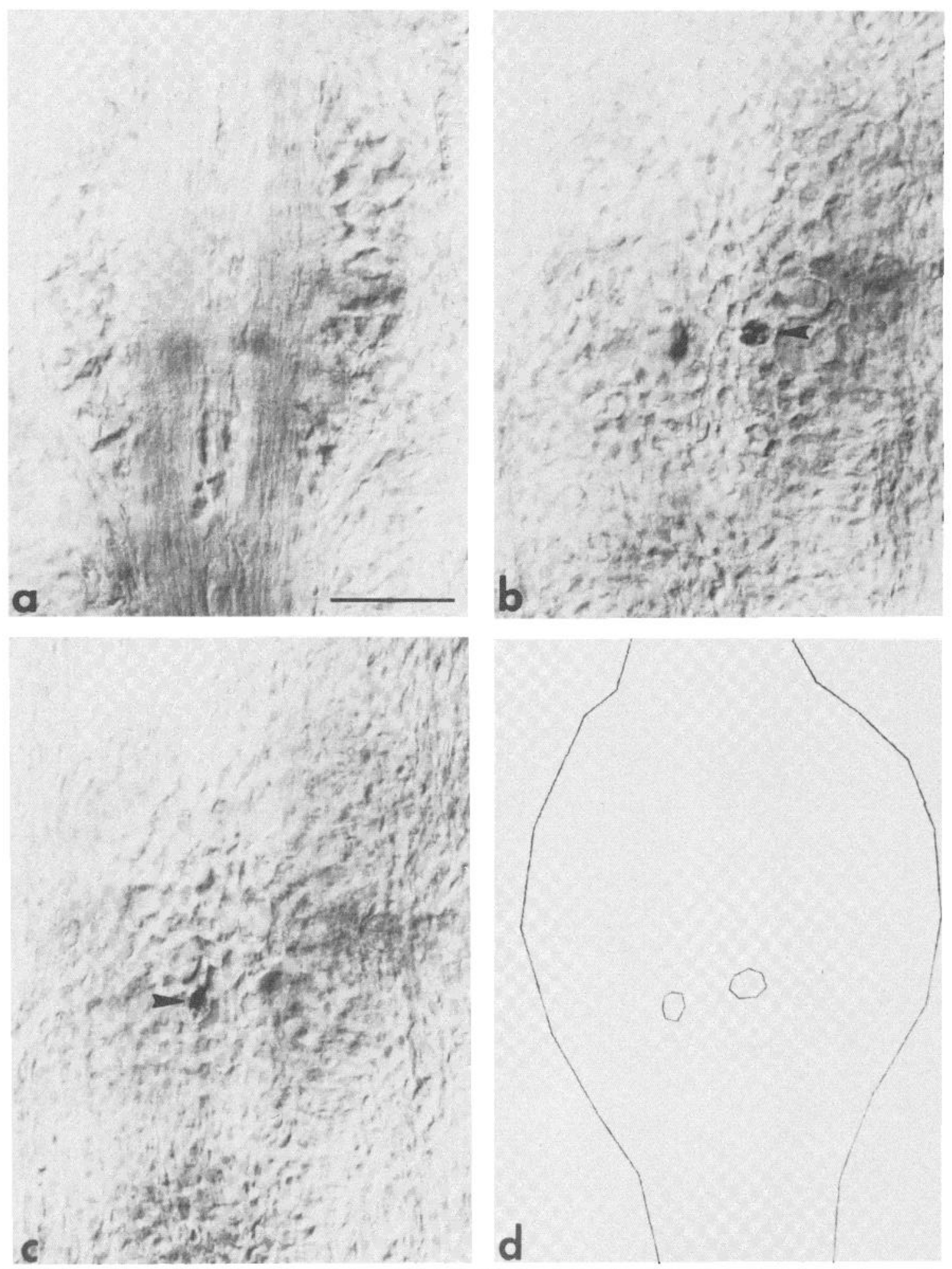

Figure 5. Micrographs of SG4 in a 10-day embryo labeled with Lan3-6. $a, b$, and $c$ show three focal planes, from dorsal to ventral. $d$ is a computer-aided drawing of the ganglionic boundary and the two neurons that show label at this stage. The labeled neurons are indicated by arrowheads $(b$ and $c)$. (These and subsequent micrographs were taken with Nomarski optics in order to highlight details of the SG, but at the expense of some ambiguity in the visualization of lightly labeled neurons. Bright-field illumination was employed in the determination of which neurons were labeled by the mAbs.) Anterior is up. Bar $=25 \mu \mathrm{m}$.

we cannot exclude at this time the possibility that accurately matched cell proliferation and cell death maintain this number the same at later times. With respect to SG5 and SG6, however, it is clear that further cell prolifera- tion must take place, since the adult has close to 700 neurons in each of these ganglia (Macagno, 1980). Preliminary cell counts in a 46-day animal (about 2 weeks post-emergence) show an increase in cell number in SG5 

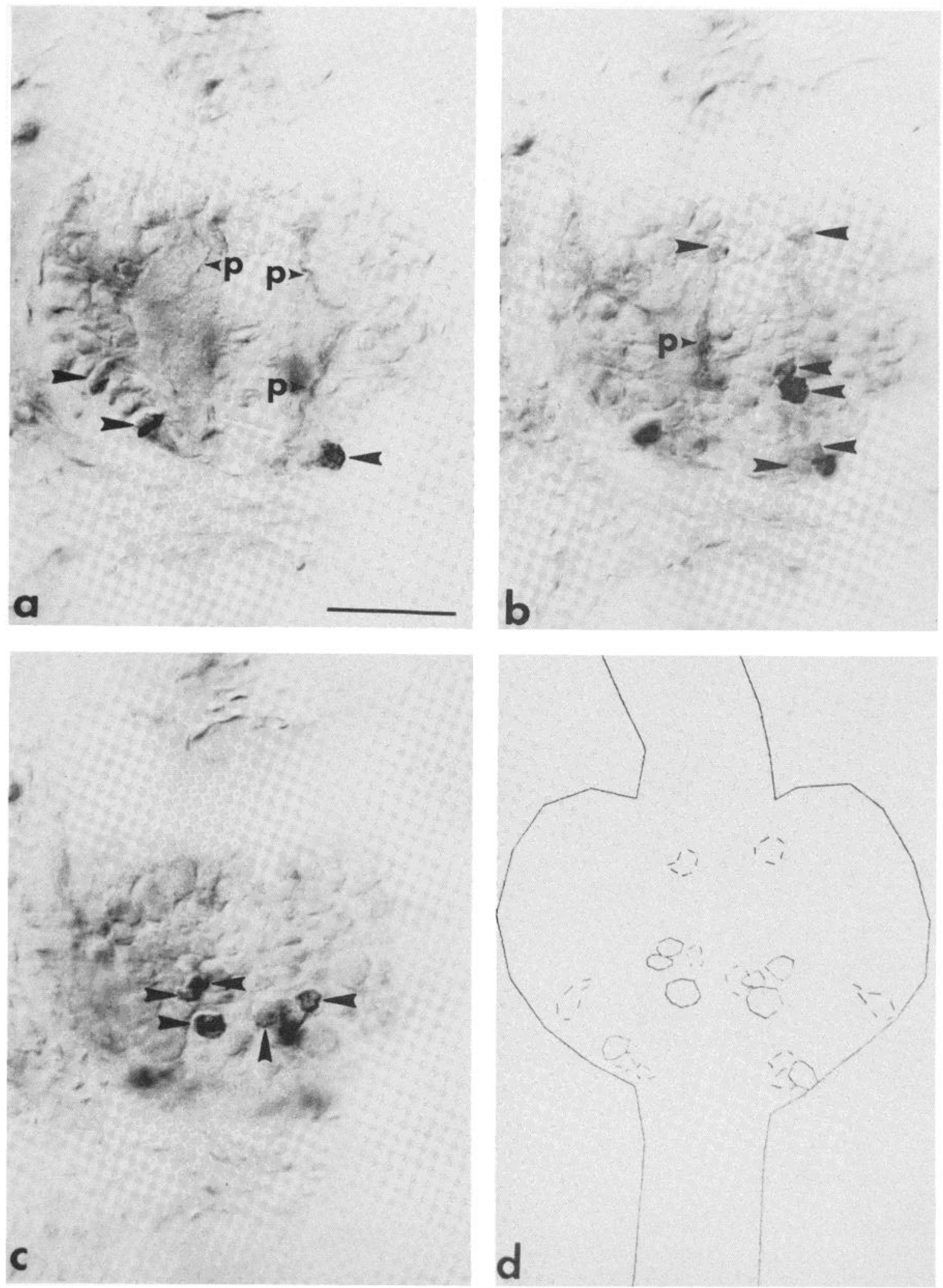

Figure 6. Micrographs of SG3 in a 13-day embryo labeled with Lan3-6. $a, b$, and $c$ show three focal planes, from dorsal to ventral. Labeled neurons in focus in these micrographs are indicated by arrowheads (some are not in focus in any of the three micrographs). Labeled processes are also indicated $(p) . d$ is a computer-aided drawing of the ganglionic boundary and the labeled neurons. Heavily labeled cells are drawn in solid lines, lightly labeled ones are drawn with dashed lines. Anterior is up. Bar $=25$ $\mu \mathrm{m}$. 

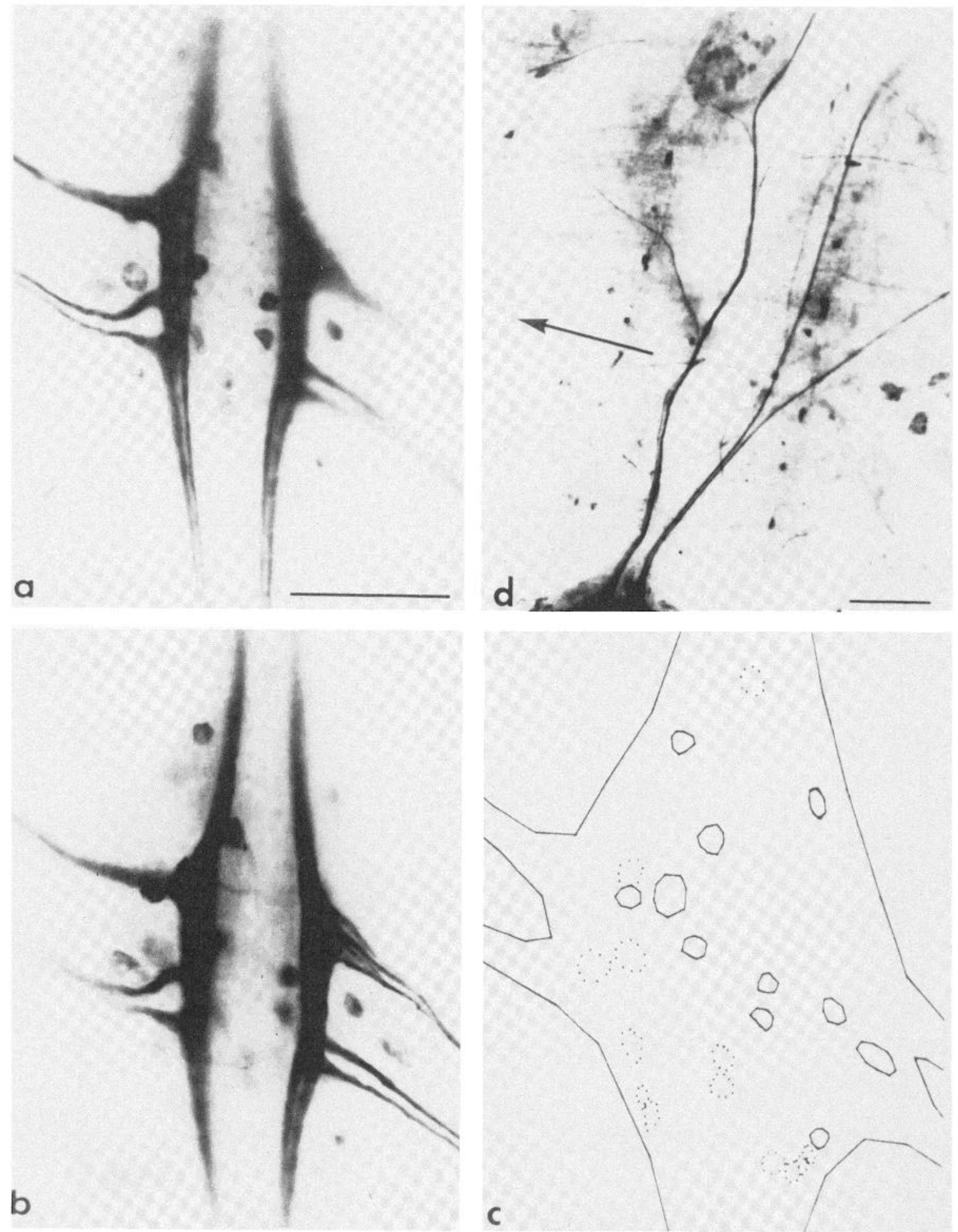

Figure 7. Labeling with Lan3-6 in a 25-day embryo. $a$ and $b$ show two focal planes of SG12. Many neurons, as well as processes in the roots, connectives, and neuropil, are labeled. $c$, Computer-aided drawing of the ganglionic boundary and labeled neurons in SG12; heavily labeled cell bodies are drawn in solid lines, lightly labeled ones are drawn in dotted lines. In $a, b$, and $c$, anterior is up. Bar in $a=100 \mu \mathrm{m}$, and applies also to $b$ and $c$. A lower power micrograph of the body wall to the right of SG8 is shown in $d$. Processes in the roots and their branches are seen to be heavily labeled by Lan3-6. The arrow points toward anterior; the bar in $d=100 \mu \mathrm{m}$.

(430 cells) and no significant change in SG10 (386 cells), indicating that the additional neurons in the sex ganglia begin to appear early postembryonically. In correlation with this possibility, when we screened Lan3-1 on 46 - day-old animals, we detected a low level of binding on the cell bodies of an additional pair of neurons in SG5 and in SG6. These were located on the ventral surface (Fig. 10), in positions similar to those found in the adult 
TABLE II

Average numbers of neurons per ganglion labeled by Lan3-6 for grouped segmental ganglia in different regions of the nerve cord and for all segmental ganglia at different times in development

The errors quoted are standard deviations of the average. The primary data appear in Table I.

\begin{tabular}{|c|c|c|c|c|c|c|c|c|}
\hline \multirow{2}{*}{$\begin{array}{l}\text { Segmental } \\
\text { Ganglion }\end{array}$} & \multicolumn{7}{|c|}{ Days of Development } & \multirow{2}{*}{ Adult } \\
\hline & $8 \mathrm{~d}$ & $9 \mathrm{~d}$ & $10 \mathrm{~d}$ & $11 \mathrm{~d}$ & $12 \mathrm{~d}$ & $13 \mathrm{~d}$ & $25 \mathrm{~d}$ & \\
\hline G1 to G5 & $1.9 \pm 0.2$ & $2.0 \pm 0$ & $2.1 \pm 0.2$ & $8.1 \pm 0.9$ & $15.6 \pm 2.0$ & $17.8 \pm 1.5$ & $18.3 \pm 2.1$ & $37 \pm 2$ \\
\hline G6 to $\mathrm{G} 10$ & $0.8 \pm 0.8$ & $1.8 \pm 0.4$ & $2.3 \pm 0.3$ & $7.0 \pm 0.9$ & $13.9 \pm 1.7$ & $21.5 \pm 2.8$ & $21.7 \pm 2.5$ & $48 \pm 6$ \\
\hline G11 to $\mathrm{G} 15$ & 0 & $0.5 \pm 0.5$ & $2.3 \pm 0.8$ & $4.3 \pm 0.8$ & $9.4 \pm 0.9$ & $16.5 \pm 0.8$ & $20.3 \pm 5.1$ & $47 \pm 4$ \\
\hline G16 to G21 & 0 & 0 & $1.0 \pm 1.0$ & $2.1 \pm 1.1$ & $4.9 \pm 2.9$ & $13.5 \pm 3.4$ & $15.7 \pm 2.5$ & $40 \pm 6$ \\
\hline $\mathrm{G} 1$ to $\mathrm{G} 21$ & $0.65 \pm 0.9$ & $1.0 \pm 0.9$ & $1.9 \pm 0.9$ & $5.2 \pm 2.6$ & $10.4 \pm 4.7$ & $17.2 \pm 3.7$ & $19.1 \pm 3.9$ & $43 \pm 6$ \\
\hline
\end{tabular}

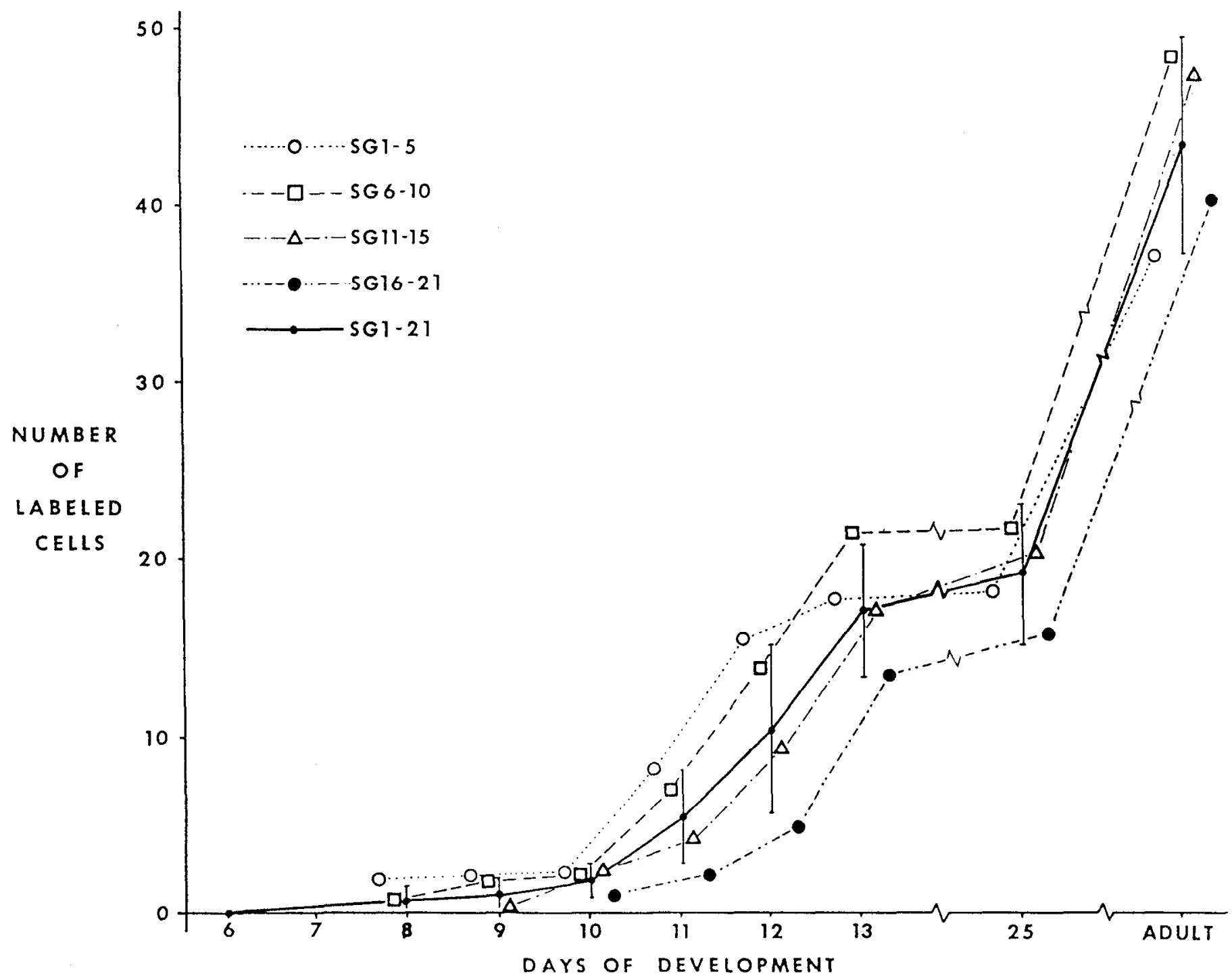

Figure 8. Graph of the average number of neurons labeled by Lan3-6 in various groups of segmental ganglia as a function of days in development. The numerical data are presented in Table II. Only the standard deviations of the averages of all ganglia (SG1-21) have been indicated in the graph. Symbols are displaced laterally for clarity.

by Zipser and McKay (1981). We do not know at present whether they are the same cells, though it would seem likely.

\section{Discussion}

The data presented show that all three mAbs raised against adult leech nerve cords label embryonic structures. The patterns of labeling have distinct spatial and temporal features which we will discuss for each $\mathrm{mAb}$ individually.

Lan3-5. The antigens recognized by this mAb are expressed in the embryo by both nervous tissue and nephridia. The uniform distribution of label throughout the nephridia suggests that Lan3-5 does not recognize neural components in this tissue. Whether the molecules that are recognized are the same or only share similar 

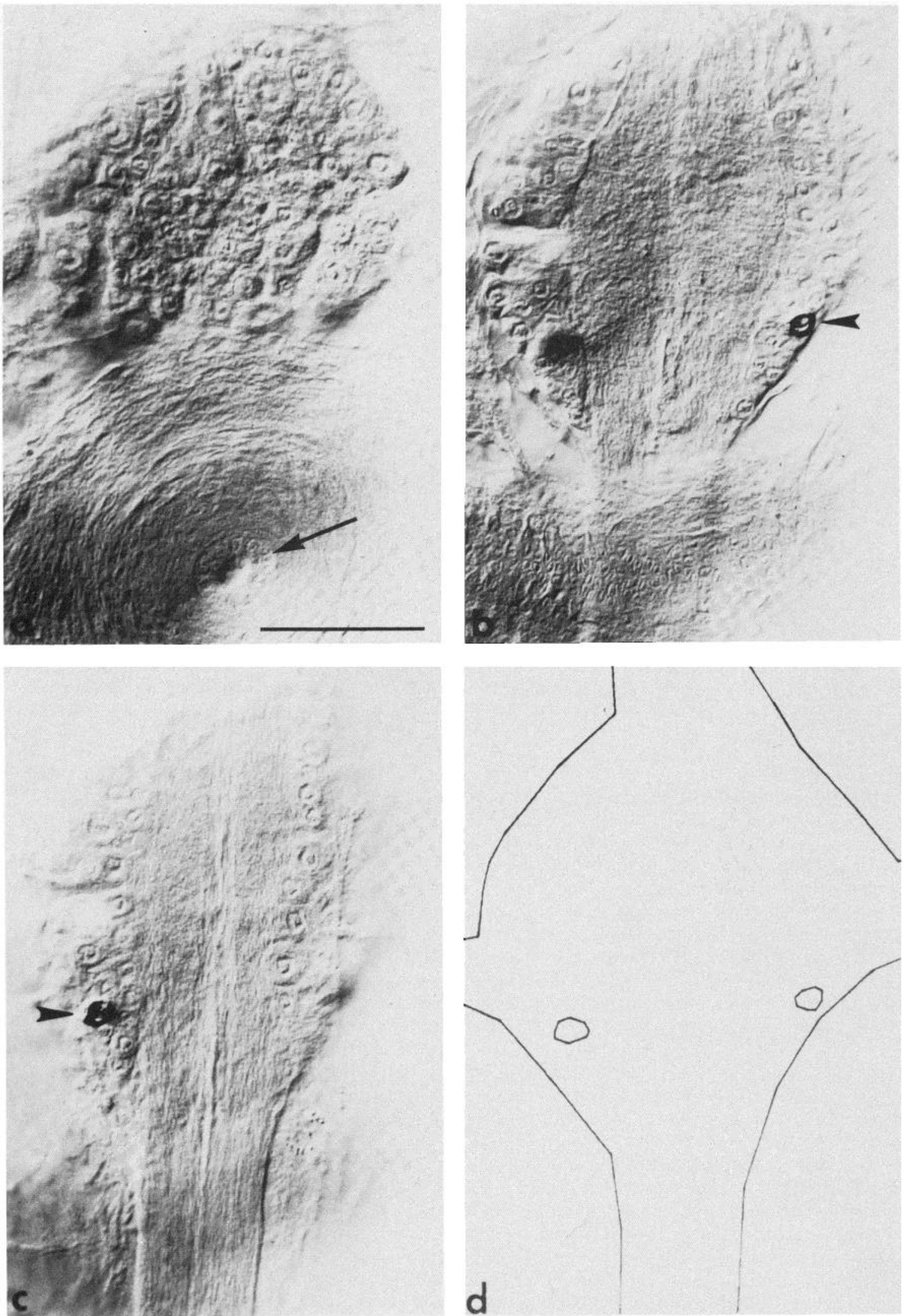

Figure 9. Micrographs of SG5 from a 20-day embryo labeled with Lan3-1. $a$, $b$, and $c$ show three focal planes from ventral to dorsal. Only two small neurons are labeled by Lan3-1 at this stage (arrowheads). The male genital pore is indicated by an arrow in $a$. $d$ shows a drawing of the ganglionic boundary and the two labeled cell bodies. Anterior is up. Bar $=50 \mu \mathrm{m}$. 


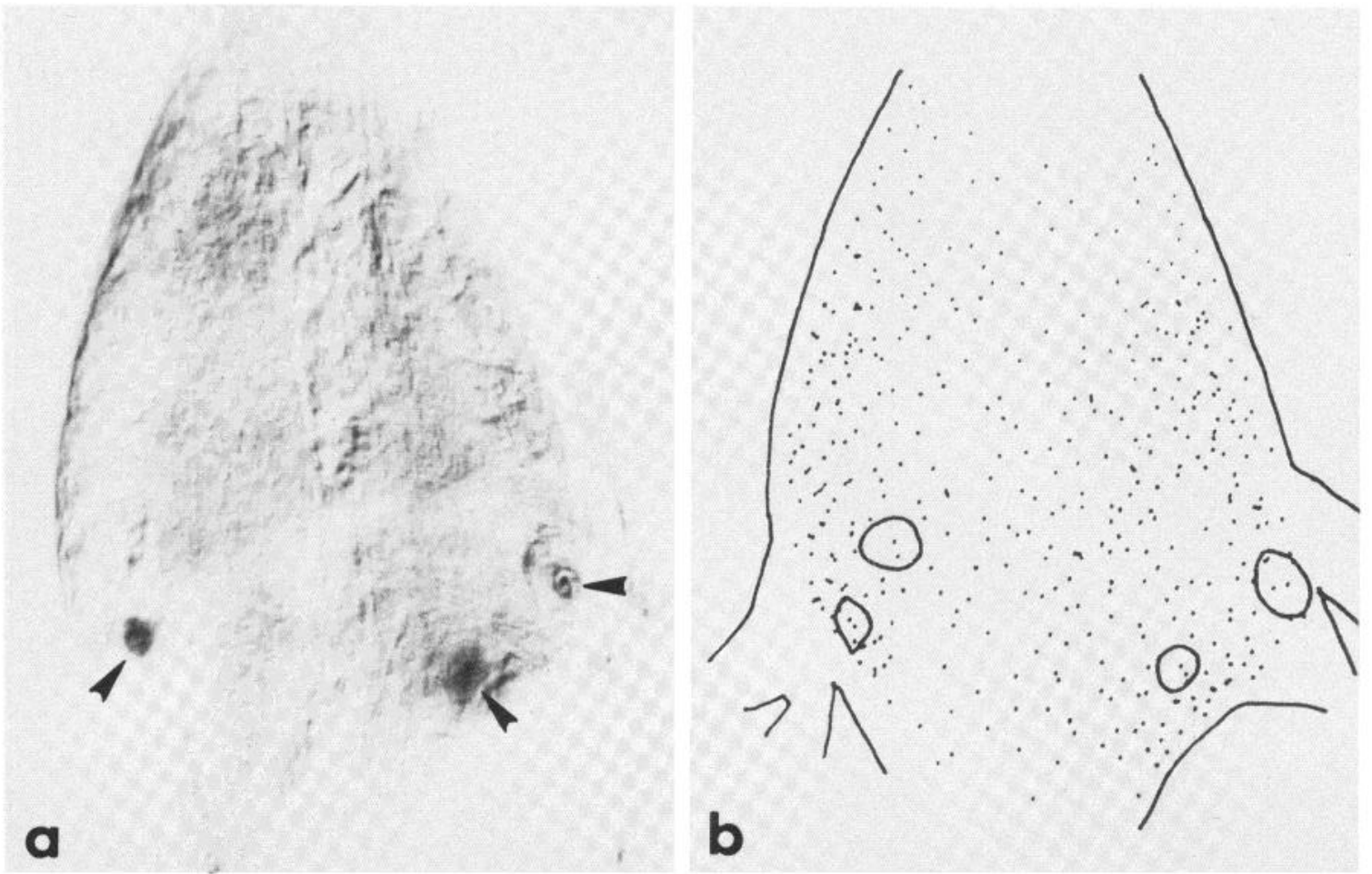

Figure 10. Labeling with Lan3-1 in a 46-day animal (2 weeks post-emergence). The ganglion shown is SG6. Four neurons are labeled, two located dorsally and two ventrally. This is the earliest stage that we find the ventral cells labeling. $a$, Micrograph of a whole mount of SG6. One of the ventrally located neurons is seen at the right (arrowhead). The label is most visible in the perinuclear region. The other labeled ventral neuron is not seen at this plane of focus. The pair of labeled dorsal neurons common to all ganglia appear out of focus (arrowheads). $b$, Computer-aided drawing of this ganglion. The four neurons labeled by Lan31 are outlined. The locations of the centers of each of the nuclei of the 430 neurons in this ganglion are indicated by points. Anterior is up.

antigenic sites is unknown, but efforts to characterize the Lan3-5 antigens are presently under way (M. Flaster and B. Zipser, personal communication). It is of interest to note that two other mAbs raised against leech CNS (Lan3-2 and Laz2-369), which bind to both nervous and gut tissues, react with multiple antigen bands on Western blots prepared from these tissues (Zipser et al., 1982; Hogg et al., 1983). In these instances the CNS neuronal antigens and the gut epithelial antigens are different. In any case, the labeling of the nephridia by Lan3-5 provides us with a useful marker for staging embryonic ganglia in a manner that is independent of the nervous system itself.

Within nervous tissue, the first structures labeled by Lan3-5 are associated with the forming roots. The distribution of label indicates that the cells that bind Lan35 are glia and that neuronal processes in the roots are not themselves labeled. It is of interest to note that the labeling with Lan3-5 allows us to identify these presumptive root glia at stages ( 6 to 7 days) when processes of central neurons are beginning to grow out to the periphery (Wallace, in Weisblat, 1981) and when peripheral neurons are beginning to grow into the CNS (unpublished observations). These glia may be playing a role in the establishment of peripheral nerves, a possibility that can be studied by labeling with Lan3-5 and dyefilling individual neurons in the same specimen. This would allow us to visualize simultaneously the axonal and glial processes to determine whether they grow at the same time or whether one precedes the other.
The labeling of the interganglionic connective glia occurs late in embryogenesis, at a time when many processes are readily recognized in the connectives. In this case, in contrast to the case of the roots, the antigens are expressed considerably later than the time when the glia first appear. Labeling of neurons by Lan3-5 also occurs late in embryogenesis, at a time when many neurons have extensive dendritic fields. The $\mathrm{P}$ cells, for example, are among the earliest to differentiate (J. Kuwada and A. P. Kramer, submitted for publication); however, they label with Lan3-5 only at the end of embryogenesis or postembryonically. No simple temporal correlation between the expression of antigens recognized by Lan3-5 and specific types of morphologically recognizable events is apparent from these initial studies.

Lan3-6. The expression by ganglionic neurons of the antigenic sites recognized by Lan3-6 is a gradual process. For a particular SG, as shown by the data in Table I, the number of neurons labeled by Lan3-6 increases monotonically with embryonic age, with about half of the number in the adult labeling at the end of embryogenesis. The significance of this should be considered in light of our preliminary observations (unpublished) concerning cell number as a function of embryonic age. We have found that SG in 10- to 12-day embryos have a significantly larger number of neurons than adult SG (except in SG5 and SG6. The number decreases to the adult value by the end of embryogenesis. This net loss could be due entirely to cel! seath or to a combination of cell 
proliferation and cell death. Whatever is the case, the number of cells labeled by Lan3-6 is increasing at the same time that a significant loss of cells (up to $15 \%$ ) is taking place in each ganglion. Although no loss of cells labeled by Lan 3-6 is clearly evident, the loss of a few such cells would probably be hidden within the variability we see from specimen to specimen. It is of interest to note that Stuart et al. (D. K. Stuart, S. S. Blair, and D. A. Weisblat, submitted for publication) have found more neurons that contain monoamines at some embryonic stages than are found in the adult. Whether this is duc to a loss of some of these neurons or to their stopping to make monoamines is not known at present.

Segmental differences are seen in both the onset of labeling with Lan3-6 and the total number of cells labeled. The time dependence seems to be a reflection of a general temporal gradient of development from anterior to posterior which is seen in both neuronal and other tissues. Segmental differences in the numbers of labeled neurons seen in the adult (see Table II) begin to appear in the middle of embryogenesis, at around 13 days of development. This seems particularly clear in the more anterior ganglia, which in the adult show fewer neurons labeled with Lan3-6 than do the ganglia in the middle of the nerve cord. This observation supports the suggestion that segmental differentiation may be an early event. However, as will be discussed below with respect to Lan31 , we have reason to believe that this hypothesis does not apply to all segmental differences.

Among the earliest neurons labeled by Lan3-6, the label is first found on the cell bodies, and only later in processes. We can not determine from our observations whether this is due to these neurons lacking processes at these times or not. However, it is the case that certain neurons that label at later times, particularly postembryonically, do so after they have undergone considerable morphological differentiation (e.g., the $\mathrm{P}$ cells). An attractive, though entirely hypothetical possibility, is that expression of the antigens by different neurons is an inductive phenomenon, occurring serially as the various neurons involved each become part of a particular functional circuit.

Lan3-1. At the embryonic stages in which the labeling of neurons with this antibody was studied, only a homologous pair of dorsal cells was found to be labeled in all ganglia. By 2 weeks after emergence, however, additional cells in the sex ganglia begin to express detectable levels of antigens bound by Lan3-1. The ventral pairs of cells labeled by Lan3-1 in ganglia 5 and 6 of adults (Zipser, 1982) are part of the additional complement of a few hundred cells that are found only in these two ganglia (Macagno, 1980). Preliminary data from cell counts at late embryonic and early postembryonic stages indicate that these additional cells do not appear in ganglia 5 and 6 until postembryonic stages. Since in one case (ganglion 6) the extra pair of cells labeled by Lan31 are known to have a function in sexual behavior (Zipser 1979a, b; Zipser and McKay, 1981), the delay in the appearance of these neurons may reflect the delay in maturation of sexually related structures in these leeches.
Whether these neurons arise from delayed mitosis of intraganglionic precursor cells or from the migration of cells into these ganglia (as seen in Aplysia by M. Jacob, submitted for publication) remains to be determined. In contrast to the segmental differences found with Lan3-6 in the embryo, the segmental specialization detected with Lan3-1 occurs postembryonically. The extra pairs of ventral neurons are labeled by Lan3-1 soon after they first appear in the sex ganglia. Thus Lan3-1 will be a useful marker for identifying and studying these specific neurons early in their differentiation.

\section{References}

Dawydoff, C. (1959) Ontogenese des annelides. C. Developpement des Hirudinees. In Traite de Zoologie. Tome V: Annelides, P. Grassé, ed., pp. 665-682, Masson et Cie, Paris.

Fernandez, J., and G. S. Stent (1983) Embryonic development of the hirudinid leech Hirudo medicinalis: Structure, development and segmentation of the germinal plate. J. Embryol. Exp. Morphol., in press.

Hockfield, S., and R. McKay (1983) Monoclonal antibodies demonstrate the organization of axons in the leech. J. Neurosci. 3: 369-375.

Hogg, N., M. Flaster, and B. Zipser (1983) Cross reactivities of monoclonal antibodies between select leech neuronal and epithelial tissues. J. Neurosci. Res. 9: 445-457.

Macagno, E. R. (1980) Number and distribution of neurons in leech segmental ganglia. J. Comp. Neurol. 190: 283-302.

Mann, K. H. (1962) Leeches (Hirudinea), Pergamon Press, New York.

McKay, R., S. Hockfield, J. Johansen, L. Kleina, and I. Thompson (1982) Monoclonal antibodies as probes of the leech nervous system. Soc. Neurosci. Abstr. 8: 714.

Stent, G. S., D. A. Weisblat, S. S. Blair, and S. L. Zackson (1982) Cell lineage in the development of the leech nervous system. In Neuronal Development, N.C. Spitzer, ed., pp. 144, Plenum Press, New York.

Stewart, R. R., E. R. Macagno, and B. Zipser (1982) The labeling patterns of developing leech CNS neurons with monoclonal antibodies raised against adult nervous tissue. Soc. Neurosci. Abstr. 8: 15.

Weisblat, D. A. (1981) Development of the nervous system. In Neurobiology of the Leech, K. J. Muller, J. G. Nicholls, and G. S. Stent, eds., pp. 173-195, Cold Spring Harbor Laboratory, Cold Spring Harbor, NY.

Zipser, B. (1979a) Identifiable neurons controlling penile eversion in the leech. J. Neurophysiol. 12: 455-464.

Zipser, B. (1979b) Voltage-modulated membrane resistance in coupled neurons. J. Neurophysiol. 42: 465-475.

Zipser, B. (1982) Complete distribution patterns of neurons with characteristic antigens in the leech central nervous system. J. Neurosci. 2: 1453-1464.

Zipser, B., and R. McKay (1981) Monoclonal antibodies distinguish neurons in the leech. Nature 289: 549-554.

Zipser, B., S. Hockfield, and R. McKay (1981) Immunological identification of specific neurons. In Neurobiology of the Leech, K. J. Muller, J. G. Nicholls, and G. S. Stent, eds., pp. 235-247, Cold Spring Harbor Laboratory, Cold Spring Harbor, NY.

Zipser, B., N. Hogg, J. Smart, and F. Hendrickson (1982) Some monoclonal antibodies which recognize leech neurons also distinguish classes of peripheral epithelium. Snc. Neurosci. Abstr. 8: 416. 